\title{
A descriptive study of transvesical prostatectomy outcomes at a general hospital in Addis Ababa, Ethiopia
}

\author{
Henok Seife, Andualem Deneke \\ Department of Surgery, College of Health Sciences, School of Medicine, Addis Ababa University, Addis Ababa, Ethiopia \\ Correspondence: Dr Henok Seife (henokyees@gmail.com)
}

(c) 2018 H. Seife \& A. Deneke. This open access article is licensed under a Creative Commons Attribution 4.0 International License (http://creativecommons.org/licenses/by/4.0/) which permits unrestricted use, distribution, and reproduction in any medium, provided you give appropriate credit to the original author(s) and the source, provide a link to the Creative Commons license, and indicate if changes were made.

\begin{abstract}
Background

Benign prostatic hyperplasia (BPH) affects more than $50 \%$ of men over 60 years of age and is the most common cause of bladder outlet obstruction and voiding symptoms in elderly men. Open prostatectomy-either transvesical or retropubic - remains the standard definitive treatment for BPH in some centres, especially in developing countries.

Methods

This study was undertaken at Ras Desta Hospital, a regional hospital run by the Addis Ababa Health Bureau. The study was conducted between 1 January 2011 and 31 December 2013, during which time 195 patients underwent surgery for BPH. Of these 154 patients had preoperative and postoperative records sufficient for comparison and analysis. Data were analysed using SPSS version 20
\end{abstract}

\begin{abstract}
Results
Fourteen patients (9\%) were between 40 and 49 years of age, 41 (27\%) were 50 to 59 years old, and 67 patients (44\%) were between 60 and 69 years of age. Sixty-one of the patients (40\%) of patients had one or more episodes of acute urinary retention and had a history of catheterization for bladder outlet obstruction. Immediate postoperative complications were seen in 10 patients. Two patients had transient incontinence after transvesical prostatectomy, and 1 patient had permanent incontinence. There was also 1 clot retention, 2 suprapubic leaks, and 4 wound infections. One patient reported retrograde ejaculation.
\end{abstract}

\section{Conclusions}

Given the limited availability and high costs associated with transurethral resection of the prostate, open prostatectomy remains an appropriate alternative in Ethiopia. The complication rates associated with open prostatectomy are minimal when the procedure is performed by adequately trained personnel. However, BPH patients often present to hospital after several serious complications. Therefore, more efforts should be made towards improving community awareness.

Keywords: benign prostatic hyperplasia, $\mathrm{BPH}$, open prostatectomy, transvesical, Ethiopia

\section{Introduction}

Benign prostatic hyperplasia (BPH) affects more than $50 \%$ of men over 60 years of age and is the most common cause of bladder outlet obstruction and voiding symptoms in elderly men. ${ }^{1,2}$ BPH is characterized by lower urinary tract symptoms (LUTS) and sometimes may be associated with urinary incontinence and sexual dysfunction. Apart from the daily misery of these symptoms, if patients seek medical advice late, they could also suffer from complications, such as uri- nary tract infection (UTI), pyelonephritis, stone formation, hydronephrosis, and even renal failure. ${ }^{3}$ Common presentations are severe LUTS, acute urinary retention (AUR), chronic urinary retention, gross haematuria and renal impairment. Many patients have indwelling urethral catheters at the time of surgery, with some even having suprapubic catheterization after failed urethral catheterization during a previous episode of acute retention. The leading indication for catheter placement is recurrent AUR and significant gross haematuria. ${ }^{4}$ 
Table 1. Postoperative complication frequency by age

\begin{tabular}{c|c|c|c|c}
\hline \multirow{2}{*}{ Age group (years) } & \multicolumn{5}{|c}{ Frequency } \\
\cline { 2 - 5 } & No complications & $\begin{array}{c}\text { Mild postoperative } \\
\text { complication managed } \\
\text { before discharge }\end{array}$ & $\begin{array}{c}\text { Transient complication } \\
\text { improved with treatment } \\
\text { after discharge }\end{array}$ & Permanent complication \\
\hline \hline $40-49$ & 13 & 0 & 0 & 1 \\
$50-59$ & 37 & 4 & 0 & 0 \\
$60-69$ & 62 & 3 & 1 & 1 \\
$\geq 70$ & 29 & 1 & 0 & 2 \\
\hline
\end{tabular}

Open prostatectomy was the operative treatment for benign prostatic enlargement for several decades until the advent of transurethral resection of the prostate (TURP), which is now the gold standard. ${ }^{5}$ In sub-Saharan Africa, however, the high cost of the required technology, limited maintenance capacity, and the absence of clear policies for the replacement of broken items limit the application of TURP to few tertiary centres. ${ }^{4}$

Eugene Fuller performed the first complete suprapubic removal of a prostatic adenoma by blind digital enucleation in $1884 .{ }^{6}$ Open prostatectomy-either via the transvesical or retropubic route-remains the reference standard for managing $\mathrm{BPH}$ in some centres, especially in developing countries. $^{7}$

Transvesical prostatectomy is associated with immediate, early, and late complications and the rates of complications vary tremendously. ${ }^{8}$ Possible immediate and early complications include bleeding, clot retention, wound infection, UTI, epididymo-orchitis, vesicocutaneous fistula, and transient incontinence. ${ }^{7,9}$ Reported overall 30 -day mortality rates have ranged between $0.9 \%$ and $2.5 \%$ following prostatectomy, with most deaths occurring within the first postoperative week, mainly due to sepsis, myocardial infarction, and pulmonary embolism. ${ }^{10}$

Some patients may turn out to have prostatic cancer during histological evaluation, and 1 study reported an $8.2 \%$ prevalence of prostatic adenocarcinoma among elderly patients who underwent transvesical open prostatectomy. ${ }^{9}$

This study aimed to describe the presentations, outcomes, complications, and biopsy results of patients who underwent transvesical prostatectomy at a general hospital in Addis Ababa, Ethiopia.

\section{Methods}

This prospective, descriptive, cross-sectional study was undertaken at Ras Desta Hospital, a regional hospital in Addis Ababa that is run by the Region 14 Health Bureau. The hospital has about 27 beds for general surgery services and 3 operating tables; 125 major surgeries are undertaken every month with only these facilities available. There were 3 general surgeons at the time of the study, but only 1 (the first author of this article) was available for the entirety of the study period (1 January 2011-31 December 2013); the other 2 surgeons were each unavailable, at separate times, for 6 consecutive months. All surgeons performed transvesical prostatectomy using a Pfannenstiel skin incision and enucleation with blunt dissection to remove the prostate. Preoperative serum prostate-specific antigen (PSA) was part of the routine workup for all patients, but only 2 of the surgeons requested histological examination of surgical biopsy samples from all prostates removed during the study period.

All patients who presented to our centre with a diagnosis of $\mathrm{BPH}$ or prostatic enlargement and were scheduled for transvesical prostatectomy during the study period were eligible for inclusion in the study. A study-specific data collection form was attached to each patient's hospital chart for gradual completion between admission and discharge; additional data were collected at follow-up appointments 1 week and 3 months after discharge. Study data were collected by doctors and nurses in the ward and the operating room, and by the operating surgeon during clinic consultations. Data were analysed using SPSS version 20 (IBM Corp., Armonk, NY, USA).

\section{Results}

During the study period, 195 patients underwent transvesical prostatectomy. Of these, sufficient preoperative and postoperative data were available for 154 patients, and this was the final number of patients included in the analysis. Fourteen patients (9\%) were between 40 and 49 years of age, 41 (27\%) were 50 to 59 years old, and 67 patients (44\%) were between 60 and 69 years of age. Addis Ababa was the city of residence of $81 \%$; the remaining $19 \%$ came from 3 different regions of Ethiopia.

Most patients (84\%) were referred from a lower-level health facility. Twenty-seven per cent of patients had to wait for less than a month for definitive surgical treatment; $36 \%$ waited for 1 to 3 months, $29 \%$ for 3 to 6 months, $12 \%$ for 6 to 12 months; 2 patients had to wait for more than a year for surgery. All patients underwent preoperative ultrasound measurement of the prostate. A volumetric report was generated via transabdominal ultrasound for 98 patients (64\%); $32 \%$ patients had an estimated prostate volume less than 50 $\mathrm{cm}^{3}, 23 \%$ had a prostate volume between 50 and $80 \mathrm{~cm}^{3}, 8 \%$ had a volume greater than $80 \mathrm{~cm}^{3}$, and volume was not determined for $34 \%$ of patients. 
Table 2. Preoperative acute urinary retention and catheterization history

\begin{tabular}{c|c|c}
\hline Preoperative catheterization status & n & $\%$ \\
\hline \hline Catheterized $\leq 1$ month before surgery & 31 & 20 \\
Catheterized for between 1 and 3 months before surgery & 15 & 10 \\
Catheterized for more than 6 months before surgery & 6 & 4 \\
Previously catheterized for acute urinary retention but catheter removed & 9 & 60 \\
No history of acute urinary retention or catheterization & 92 & 6 \\
\hline
\end{tabular}

\begin{tabular}{|c|c|c|}
\hline Complication & $\mathbf{n}$ & $\%$ \\
\hline No complications & 125 & 81 \\
\hline Hydronephrosis & 11 & 7 \\
\hline $\begin{array}{l}\text { Obstructive uropathy with } \\
\text { derangment of renal function tests }\end{array}$ & 7 & 5 \\
\hline Chronic cystitis & 7 & 5 \\
\hline Other & 4 & 3 \\
\hline Total & 154 & \\
\hline
\end{tabular}

Sixty-one patients (39\%) had 1 or more episodes of acute urinary retention and a history of catheterization for bladder outlet obstruction. Among these, 9 had only been transiently catheterized, 31 had been catheterized for less than 1 month, 15 for 1 to 3 months, and 6 patients had indwelling catheters for more than 3 months before surgery.

Preoperative evaluation revealed $19 \%$ of patients to have 1 or more chronic obstructive symptoms of bladder outlet obstruction. Eighteen patients had obstructive uropathy, among whom 11 had mild to moderate hydronephrosis, and 7 had severe hydronephrosis with renal function derangement. Other chronic features included chronic cystitis in 7 patients and diverticula and trabeculations observed in 4 patients. No bladder stones were observed in our patients.

Out of the 154 patients operated upon, 148 (96\%) had confirmed BPH, 5 (3\%) had bladder neck stenosis, and 1 had a posterior urethral stricture. The stricture was an intraoperative finding and managed accordingly.

Postoperative complications were evaluated, and 2 patients had transient postoperative incontinence, and there were no cases of permanent incontinence. Only 1 patient reported retrograde ejaculation after surgery. A total of 4 wound infections occurred, 2 of which were superficial and managed conservatively. One patient required readmis- sion for delayed wound infection, which was managed with wound care. One patient had to stay in the hospital for more than 1 week because of a wound infection; this patient underwent secondary wound closure for partial surgical wound dehiscence. One patient experienced postoperative clot retention and required re-exploration to achieve haemostasis; this patient recovered well thereafter and required transfusion of 2 units of whole blood. None of the other patients required transfusion. Suprapubic irrigation catheters were applied to all patients, and 2 patients developed postoperative transient suprapubic urinary leakage and fistula formation, as diagnosed by the presence of leaking urine after removal of the suprapubic catheter. Both of these patients recovered after strapping and continuous irrigation of the transurethral catheter. There were no deaths reported during admission or follow-up.

Most patients (58\%) stayed in the hospital for 3 to 4 days postoperatively; $22 \%$ of patients were admitted for 5 to 7 days after surgery. Ten per cent of patients were discharged within 3 postoperative days and had no complications recorded on follow-up. Ten per cent of patients needed to stay for more than 7 days, most of whom had 1 or more of postoperative complications.

Of the 154 patients operated upon, biopsy results were returned for 83 patients (54\%); these are the patients for whom PSA results and cancer incidence are reported herein. Four of these 83 patients (5\%) had prostate cancer. Among the 79 patients whose histology findings were negative for cancer, 26 had a PSA below $4 \mathrm{ng} / \mathrm{mL}$, and 12 had a PSA between 8 and $12 \mathrm{ng} / \mathrm{mL}$. Twenty-two patients had PSA values between 4 and $8 \mathrm{ng} / \mathrm{mL}, 1$ of whom had histologically confirmed prostate cancer. Eleven patients had PSA values between 12 and $30 \mathrm{ng} / \mathrm{mL}$, among whom 1 biopsy sample was positive for cancer. Four patients had PSA values greater than $30 \mathrm{ng} / \mathrm{mL}$, and 2 of these had histologically confirmed prostate cancer.

In terms of postoperative complications, the incidence was highest in the 50- to 59-year age group ( 4 of $41 ; 10 \%$ ). Of 14 patients in the 40 - to 49 -year age group, there was 1 postoperative complication (7\%). Among 67 patients between 60 and 69 years of age, there were 5 postoperative complications (7\%). Among 32 patients 70 years old and above, 3 (9\%) experienced postoperative complications. 
Table 4. Frequency table of serum prostate-specific antigen (PSA) results by prostate biopsy findings

\begin{tabular}{|c|c|c|c|c|c|c|}
\hline \multirow{2}{*}{ Biopsy result } & \multicolumn{6}{|c|}{ PSA result } \\
\hline & $<4$ & 4-8 & 8-12 & $12-30$ & $>30$ & Not determined \\
\hline Benign prostatic hyperplasia & 26 & 22 & 12 & 11 & 4 & 4 \\
\hline Malignant prostatic cancer & 0 & 1 & 0 & 1 & 2 & 0 \\
\hline Not documented & 26 & 16 & 13 & 7 & 2 & 7 \\
\hline Total & 52 & 39 & 25 & 19 & 8 & 11 \\
\hline
\end{tabular}

\section{Discussion}

The majority of our patients were between 60 and 69 years of age, which is similar to another study from Ethiopia ${ }^{3}$; an open prostatectomy study from Nigeria reported the commonest age range to be 70 to 79 years. ${ }^{5}$ Research from Ethiopia ${ }^{3}$ and Nigeria $^{4}$ has reported the starting age of symptomatic $\mathrm{BPH}$ requiring surgery to be in the fifth decade of life, while other studies from Nigeria ${ }^{5}$ and Brazil ${ }^{1}$ observed symptom onset in the sixth decade.

Many patients (32\%) in our study sample had a prostate that was less than $50 \mathrm{~g}$, a finding that is similar to other Ethiopian studies, ${ }^{3,12}$ whereas in studies from Brazil ${ }^{1}$ and Nigeria, ${ }^{4}$ all prostates were larger than $70 \mathrm{~g}$.

Sixty-one of our patients (39\%) had acute urinary retention at presentation, while previous studies in Ethiopia reported acute urinary retention rates of $78.5 \%$ and $84.4 \%$. $^{3,12}$ This discrepancy may be related to selection criteria or disparities in health service access or medical care-seeking behaviour among study sites. Two studies from Nigeria ${ }^{4,5}$ and 1 from Pakistan ${ }^{2}$ reported acute urinary retention rates of $35.6 \%, 49 \%, 26.2 \%$, respectively, which are similar to our study.

We observed upper tract obstructive uropathy (hydronephrosis) in 18 patients (12\%), among whom 7 (5\%) had severe hydronephrosis. This complication was also reported in other studies, with prevalences ranging from $6.2 \%$ to $23.4 \%$. $^{2,4,5,9}$

Immediate postoperative complications were seen in 10 of our patients (6\%), which is a much lower frequency than what has been previously reported in Ethiopia, Nigeria, and Egypt.,11,12 Immediate complications included 1 clot retention, 2 cases of transient incontinence, 2 suprapubic leaks, and 4 wound infections in our study. Only one patient required blood transfusion.

We submitted 83 surgical specimens (54\%) for histological examination. Our prostatic cancer prevalence (5\%) was lower than that $(8.2 \%)$ reported from a study carried out in Pakistan. ${ }^{2}$ A histological cancer diagnosis was rare, even if PSA was above $4 \mathrm{ng} / \mathrm{mL}$. Notably, however, all 4 cases of histologically confirmed prostatic cancers were patients whose PSAs were higher than $4 \mathrm{ng} / \mathrm{mL}$.
Ten of our patients (6\%) were misdiagnosed, with postoperative diagnoses of prostatic cancer $(n=4)$, bladder neck stenosis $(n=5)$, and urethral stricture $(n=1)$. This reinforces the importance of improving diagnostic capacity in our setting, particularly in terms of investigations and endoscopic evaluations.

Most patients (91\%) remained in the hospital for less than 1 week after surgery, which is similar with to what can be found in surgical literature originating from Europe. ${ }^{11}$ About a tenth of our patients were admitted for more than 7 days, which is beyond the usually accepted duration.

\section{Conclusions}

Given the limited availability and high costs associated with TURP, open prostatectomy remains an appropriate and safe option in Ethiopia. The complication rates associated with open prostatectomy are minimal when the procedure is performed by adequately trained personnel. Age by itself cannot be a contraindication for transvesical open prostatectomy, rather the performance status of the patient should be considered as a key criterion. PSA values might not be a direct indicator or confirmatory test for prostatic cancer, but those patients with high PSAs should be evaluated carefully. Complications in the upper urinary tract are common in patients with bladder outlet obstruction secondary to $\mathrm{BPH}$, especially those who present to hospital after several serious complications have occurred. Therefore, more efforts should be made towards improving community awareness.

\section{Competing interests}

Both authors declare that they have no competing interests related to this work.

\section{References}

1. Carneiro A, Sakuramoto P, Wroclawski ML, Forseto PH, Den Julio A, Bautzer CR, et al. Open suprapubic versus retropubic prostatectomy in the treatment of benign prostatic hyperplasia during resident's learning curve: a randomized controlled trial. Int Braz J Urol. 2016 Mar-Apr;42(2):284-92. doi: 10.1590/S1677-5538. IBJU.2014.0517.

2. Shamim M, Idrees M, Bano S. Outcome of open prostatectomy. J Surg Pak Int. 2012 Jul-Sep;17(3):87-92. 
3. Berhanu NA. The safety and efficacy of trans-vesical prostatectomy done at a primary general hospital setting in Ethiopia. East Cent Afr J Surg. 2008;13(2):53-60.

4. Ugwumba FO, Ozoemena OF, Okoh AD, Echetabu KN, Mbadiwe OM. Transvesical prostatectomy in the management of benign prostatic hyperplasia in a developing country. Niger J Clin Pract. $2014 \quad$ Nov-Dec;17(6):797-801. doi: 10.4103/1119-3077.144402.

5. Ahmed Gadam 1, Nuhu A, Aliyu S. Ten-year experience with open prostatectomy in Maiduguri. ISRN Urol. 2012;2012:406872. doi: 10.5402/2012/406872. Epub 2012 Nov 27.

6. Tubaro A, de Nunzio. The current role of open surgery in BPH. EAU-EBU Update Ser. 2006 Oct;4(5)191-201. doi: 10.1016/j. eeus.2006.07.002
7. Oranusi CK, Nwofor A, Oranusi IO Complication rates of open transvesical prostatectomy according to the ClavienDindo classification system. Niger J Clin Pract. 2012 Jan-Mar;15(1):34-7. doi: 10.4103/1119-3077.94094.

8. Ferretti M, Phillips J. Prostatectomy for benign prostate disease: open, laparoscopic and robotic techniques. Can J Urol. 2015 Oct;22 Suppl 1:60-6.

9. Ali L, Orakzai N, Ali M. Transvesical prostatectomy in elderly patients: an experience at Saidu Teaching Hospital Swat, Ann Pak Inst Med Sci. 2008;4(3): 148-51.

10. Kiptoon DK, Magoha GA, Owillah FA. Early postoperative outcomes of patients undergoing prostatectomy for benign prostatic hyperplasia at Kenyatta National Hospital, Nairobi. East Afr Med J. 2007 Sep;84(9 Suppl):S40-4
11. Elshal AM, El-Nahas AR, Barakat TS, Elsaadany MM, El-Hefnawy AS. Transvesical open prostatectomy for benign prostatic hyperplasia in the era of minimally invasive surgery: perioperative outcomes of a contemporary series. Arab J Urol. 2013 Dec;11(4):362-8. doi: 10.1016/j.aju.2013.06.003. Epub 2013 Jul 31

12. Ahmed AA. Transvesical prostatectomy in Tikur Anbessa Hospital, Addis Ababa. East Afr Med J. 1992 Jul;69(7):378-80. 